\title{
Minimization of Negative Log Partial Likelihood Function Using Reproducing Kernel Hilbert Space
}

\author{
Nur'azah Abdul Manaf ${ }^{1,2}$, Ibragimov Gafurjan ${ }^{2}$ \& Mohd. Rizam Abu Bakar ${ }^{2}$ \\ ${ }^{1}$ Malaysia-Japan International Institute of Technology, Universiti Teknologi Malaysia, KualaLumpur, Malaysia \\ 2 Department of Mathematics, Universiti Putra Malaysia, Serdang, Selangor Darul Ehsan, Malaysia \\ Correspondence: Nur'azah Abdul Manaf, Malaysia-Japan International Institute of Technology, Universiti \\ Teknologi Malaysia, KualaLumpur, Malaysia. Tel: 60-122-906-329. E-mail:azahmanaf@gmail.com
}

Received: September 22, 2013

Accepted: October 31, $2013 \quad$ Online Published: January 22, 2014

doi:10.5539/mas.v8n1p140

URL: http://dx.doi.org/10.5539/mas.v8n1p140

\begin{abstract}
Reproducing kernel Hilbert space (RKHS) can be used to estimate values of functions, derivatives and integrals of models. The RKHS kernels are useful in finding the optimizer $f(s)=\sum_{i} a_{i} K(s, t)$ of the general Cox regression model. The procedure in the minimization of the negative log partial likelihood function is being demonstrated in this paper. Partial differentiation of the loss function is performed to determine the optimal values of $f(s)$.
\end{abstract}

Keywords: partial derivatives, reproducing kernel method, Cox regression model

\section{Introduction}

The theory of reproducing kernel is a powerful instrument in many areas of mathematical research. See, for example, Aronszajn (1950), Hille (1972), Burbea (1976), Wahba (1998), Berlinet et al. (2003), Li et al. (2003). Many researchers had shown that there exist strong connection between the problems of applied sciences, mathematical analysis, and many areas of engineering. Many statistical problems can also be solved and data can be analyzed using models related to reproducing kernel Hilbert space (RKHS). RKHS enables us to estimate a variety of mathematical models. A simple linear kernel was used by Li et al. (2003) in Cox regression models to relate expression profiles of censored cancer data sets. N. Abdul Manaf et al. (2011) generated a new kernel to determine the function $f(s)=\sum_{i} a_{i} K\left(s, s_{i}\right)$ of the general Cox model and utilized partial derivatives of the negative log partial likelihood to find the optimal values for the HIV patients survival data.

\subsection{Reproducing Kernel Hilbert Space (RKHS)}

On a domain $T s$, let $H s$ be a Hilbert space such that there exists an element $\mu_{t} \in H_{s}$ for every $t \in T_{s}$ and the inner product in $H_{s}$ is $f(t)=<\mu_{t}, f>$, for every $f \in H_{s}$. Let $K(s, t)=<\mu_{s}, \mu_{t}>$. Then, $K$ is a positive definite kernel on $T_{s} \times T_{s}$, meaning that $\sum_{i, j} a_{i} a_{j} K\left(t_{i}, t_{j}\right) \geq 0$ for every $t_{1}, t_{2}, \ldots, t_{n} \in T_{s}$ and $K$ is the reproducing kernel for $H_{s}$. Inner product $\langle K(t, \cdot), K(s, \cdot)\rangle \equiv K(s, t)$ gives the originality of "reproducing kernel".

\subsection{Moore-Aronszajn Theorem}

The Moore-Aronszajn theorem mentioned by Berlinet and Thomas-Aqnan (2003) states that every kernel $K$ which is symmetric and positive definite on a set $T_{s}$ defines an incomparable RKHS (Berlinet \& Thomas-Aqnan, 2003).

The following process shows the construction of space $H_{s}$ (Berlinet \& Thomas-Aqnan, 2003).

Let $K_{s}=K\left(s, s_{i}\right)$ for all $\mathrm{s}$ in $T_{s}$. Let $H_{0}$ be the linear span of $\left\{K_{s}: s \in T_{s}\right\}$. Suppose the dot product on $H_{0}$ is defined as

$$
\left\langle\sum_{i=1}^{m} a_{i} K_{s_{i}}, \sum_{j=1}^{n} b_{j} K_{y_{j}}\right\rangle=\sum_{i=1}^{m} \sum_{j=1}^{n} \overline{a_{i}} b_{j} K\left(y_{j}, s_{i}\right) .
$$


The symmetry of $K$ generates the symmetry of the dot product.

If we let $H_{s}$ be the complete set of $H_{0}$ with respect to the above dot product, then $H_{\mathrm{s}}$ is the compilation of functions

$$
f(s)=\sum_{i=1}^{\infty} a_{i} K_{s_{i}}(s)
$$

We observed from the Cauchy-Schwarz inequality that this series converges for every $x$. We obtain the inner product

$$
\left\langle f, K_{s}\right\rangle=\left\langle\sum_{i=1}^{\infty} a_{i} K_{s_{i}}, K_{s}\right\rangle=\sum_{i=1}^{\infty} a_{i} K\left(s_{i}, s\right)=f(s) .
$$

from the reproducing kernel properties.

\subsection{Kernel Method and Its Application}

Manaf et al. (2011) used $K(x, y)=<C x, D y>$ to obtain $f(x)$ for the general Cox model

$$
\lambda_{i}\left(t \mid x_{i}\right)=\lambda_{0}(t) \exp \left(f\left(x_{i}\right)\right)
$$

A regularized formulation of Cox regression, $R_{\text {reg }}(f)$ was considered as a problem in terms of RKHS, $H_{K}$, and

$$
\min R_{\text {reg }}(f)=\frac{1}{n} \sum_{i=1}^{n} V\left(t_{i}, \delta_{i}, f\left(x_{i}\right)\right)+\xi\|f\|_{H_{K}}^{2}
$$

where $V\left(t_{i}, \delta_{i}, f\left(x_{i}\right)\right)$ is the loss function which depends on $f(x)$ at points $\left\{f\left(x_{i}\right)\right\}_{i=1}^{n}$. The norm defined in $H_{K}$ is denoted by $\|f\|^{2}$ where $f=b+h, h \in H_{k}, b \in \Re$, and $\xi>0$ is a tuning parameter. For the general Cox model, $t_{i}$ is the survival time when $\delta_{i}=1$, and is the censoring time when $\delta_{i}=0$.

The use of negative log partial likelihood function in the regression model

$$
\lambda_{i}\left(t \mid x_{i}\right)=\lambda_{0}(t) \exp \left(f\left(x_{i}\right)\right)
$$

leads us to find the function $f(x)$ that minimizes

$$
R_{\text {reg }}(f)=-\frac{1}{n} \sum_{i=1}^{n} \delta_{i}\left[f\left(x_{i}\right)-\log \left\{\sum_{j \in R_{i}} \exp \left(f\left(x_{i}\right)\right\}\right]+\xi\|f\|_{H_{k}}^{2},\right.
$$

where $R_{i}=\left\{j: t_{j} \geq t_{i}, j=1,2, \ldots, n\right\}$ is the set of HIV patients who were at risk at $t_{i}$. The solution of this problem was given by Kimeldorf and Wahba and is known as the representer theorem in which the optimizer function $f(s)$ has the form (Kimeldorf \& Wahba, 1971)

$$
f(s)=c+\sum_{i=1}^{n} a_{i} K\left(s, s_{i}\right),
$$

where $K$ is the reproducing kernel of $H_{k}$. Constant c can be omitted in the solution procedure because it can be absorbed into baseline hazard function.

\section{Formulas for Partial Derivatives of Loss Function} Our task is to find the function $f(x)=\sum_{i=1}^{n} a_{i} K\left(x, x_{i}\right)$ when the optimal values of vector $a=\left(a_{1}, a_{2}, \ldots, a_{n}\right)$ are
applied. We need to minimize

$$
R_{r e g}(f)=-\frac{1}{n} \sum_{i=1}^{n} \delta_{i}\left[f\left(x_{i}\right)-\log \left\{\sum_{j \in R_{i}} \exp \left(f\left(x_{j}\right)\right)\right\}\right]+\xi\|f\|_{H_{k}}^{2}
$$

which is equivalent to minimizing of

$$
-\frac{1}{n} \sum_{i=1}^{n} \delta_{i} f\left(x_{i}\right)+\frac{1}{n} \sum_{i=1}^{n} \delta_{i} \log \left\{\sum_{j \in R_{i}} \exp \left(f\left(x_{j}\right)\right)\right\}+\xi\|f\|_{H_{k}}^{2}
$$

where $R_{i}=\left\{j: t_{j} \geq t_{i}, j=1,2, \ldots, n\right\}$ is the set of individuals who were at risk at time $t_{i}$.

We can state the negative log-likelihood function as follows: 


$$
\begin{aligned}
R_{\text {reg }}(f) & =-\frac{1}{n} \sum_{i=1}^{n} \delta_{i}\left[f\left(x_{i}\right)-\ln \left\{\sum_{j \in R_{i}} \exp \left(f\left(x_{j}\right)\right)\right\}\right]+\xi\|f\|_{H_{k}}^{2} \\
& =-\frac{1}{n} \sum_{i=1}^{n} a_{i}\left(\sum_{j=1}^{n} \delta_{j} K\left(x_{j}, x_{i}\right)\right)+\frac{1}{n} \sum_{i=1}^{n} \delta_{i} \ln \left\{B_{1(i)}\right\}+\xi \sum_{i=1}^{n} a_{i}\left(\sum_{j=1}^{n} a_{j} K\left(x_{i}, x_{j}\right)\right) .
\end{aligned}
$$

$R_{\text {reg }}(f)$ is minimized by using the Newton-Raphson method. We give an illustrative example for $R_{i}$. Let $n=4$ and $t_{1}=1, t_{2}=5, t_{3}=6, t_{4}=8$. Then

$R_{1}=\left\{j: t_{j} \geq t_{1}, j=1,2,3,4\right\}=\{1,2,3,4\}$,

$R_{2}=\left\{j: t_{j} \geq t_{2}, j=1,2,3,4\right\}=\{2,3,4\}$,

$R_{3}=\left\{j: t_{j} \geq t_{3}, j=1,2,3,4\right\}=\{3,4\}$,

$R_{4}=\left\{j: t_{j} \geq t_{4}, j=1,2,3,4\right\}=\{4\}$.

Let $A=-\frac{1}{n} \sum_{j=1}^{n} \delta_{j} f\left(x_{j}\right)$, the first term of (2). Then

$$
A=-\frac{1}{n} \sum_{i=1}^{n} \sum_{j=1}^{n} \delta_{j} a_{i} K\left(x_{j}, x_{i}\right)=-\frac{1}{n} \sum_{i=1}^{n} a_{i}\left(\sum_{j=1}^{n} \delta_{j} K\left(x_{j}, x_{i}\right)\right)
$$

We obtain from (3) that

$$
\frac{\partial A}{\partial a_{p}}=-\frac{1}{n} \sum_{j=1}^{n} \delta_{j} K\left(x_{j}, x_{p}\right), p=1, \ldots, n .
$$

and

$$
\frac{\partial^{2} A}{\partial a_{p} \partial a_{q}} \equiv 0, p=1, \ldots, n
$$

Let the second term of (2) be denoted by

$$
B=\frac{1}{n} \sum_{i=1}^{n} \delta_{i} \ln \left\{\sum_{j \in R_{i}} \exp \left(f\left(x_{j}\right)\right)\right\} .
$$

Then by definition of $f\left(x_{i}\right)$ we get

$$
B=\frac{1}{n} \sum_{i=1}^{n} \delta_{i} \ln \left\{\sum_{j \in R_{i}} \exp \left(\sum_{k=1}^{n} a_{k} K\left(x_{j}, x_{k}\right)\right)\right\}=\frac{1}{n} \sum_{i=1}^{n} \delta_{i} \ln B_{1(i)}
$$

where

$$
B_{1(i)}=\sum_{j \in R_{i}} \exp \left(\sum_{k=1}^{n} a_{k} K\left(x_{j}, x_{k}\right)\right) .
$$

Find partial derivatives of $B$. We have from (6) that

$$
\frac{\partial B}{\partial a_{p}}=\frac{1}{n} \sum_{i=1}^{n} \delta_{i} \frac{1}{B_{1(i)}} \cdot \frac{\partial B_{1(i)}}{\partial a_{p}}=\frac{1}{n} \sum_{i=1}^{n} \delta_{i} \frac{1}{B_{1(i)}} \cdot \sum_{j \in R_{i}} K\left(x_{j}, x_{p}\right) \exp \left(\sum_{k=1}^{n} a_{k} K\left(x_{j}, x_{k}\right)\right), p=1,2, \ldots, n .
$$

Denote

$$
B_{2 p(i)}=\frac{\partial B_{1(i)}}{\partial a_{p}}=\sum_{j \in R_{i}} K\left(x_{j}, x_{p}\right) \exp \left(\sum_{k=1}^{n} a_{k} K\left(x_{j}, x_{k}\right)\right)
$$

Then, clearly 


$$
\frac{\partial B}{\partial a_{p}}=\frac{1}{n} \sum_{i=1}^{n} \delta_{i} \frac{B_{2 p(i)}}{B_{1(i)}},
$$

and so

$$
\frac{\partial^{2} B}{\partial a_{p} \partial a_{q}}=\frac{\partial}{\partial a_{q}}\left(\frac{\partial B}{\partial a_{p}}\right)=\frac{\partial}{\partial a_{q}} \sum_{i=1}^{n} \delta_{i} \frac{B_{2 p(i)}}{B_{1(i)}}=\frac{1}{n} \sum_{i=1}^{n} \delta_{i} \cdot \frac{B_{1(i)} \cdot \frac{\partial B_{2 p(i)}}{\partial a_{q}}-B_{2 p(i)} \cdot \frac{\partial B_{1(i)}}{\partial a_{q}}}{B_{1(i)}^{2}}
$$

This gives

$$
\frac{\partial^{2} B}{\partial a_{p} \partial a_{q}}=\frac{1}{n} \sum_{i=1}^{n} \delta_{i}\left(\frac{1}{B_{1(i)}} \sum_{j \in R_{i}} K\left(x_{j}, x_{p}\right) K\left(x_{j}, x_{q}\right) \exp \left(\sum_{k=1}^{n} a_{k} K\left(x_{j}, x_{k}\right)\right)-\frac{B_{2 p(i)} B_{2 q(i)}}{B_{1(i)}^{2}}\right), p, q=1,2, \ldots, n,
$$

where $B_{2 q(i)}=\frac{\partial B_{1(i)}}{\partial a_{q}}$.

Let $C=\xi\|f\|_{H_{k}}^{2}$. Then by definition of $f$ we obtain

$$
C=\xi \sum_{i=1}^{n} \sum_{j=1}^{n} a_{i} a_{j} K\left(x_{i}, x_{j}\right)=\xi \sum_{i=1}^{n} a_{i}\left(\sum_{j=1}^{n} a_{j} K\left(x_{i}, x_{j}\right)\right)=\xi\left(\sum_{\substack{i=1 \\ i \neq p}}^{n} a_{i}\left(\sum_{j=1}^{n} a_{j} K\left(x_{i}, x_{j}\right)\right)+a_{p} \sum_{j=1}^{n} a_{j} K\left(x_{p}, x_{j}\right)\right)
$$

Hence

$$
\frac{\partial C}{\partial a_{p}}=\xi\left(\sum_{i=1, i \neq p}^{n} a_{i} K\left(x_{i}, x_{p}\right)+\sum_{j=1}^{n} a_{j} K\left(x_{p}, x_{j}\right)+a_{p} K\left(x_{p}, x_{p}\right)\right) .
$$

This can be written as follows:

$$
\frac{\partial C}{\partial a_{p}}=\xi\left(\sum_{\substack{i=1 \\ i \neq p}}^{n} a_{i} K\left(x_{i}, x_{p}\right)+\sum_{j=1}^{n} a_{j} K\left(x_{p}, x_{j}\right)\right)
$$

and hence

$$
\frac{\partial^{2} C}{\partial a_{p} \partial a_{q}}=\xi\left(K\left(x_{q}, x_{p}\right)+K\left(x_{p}, x_{q}\right)\right)=2 \xi K\left(x_{p}, x_{q}\right)
$$

since $K\left(x_{p}, x_{q}\right)=K\left(x_{q}, x_{p}\right)$.

\section{Result and Discussion}

Let $K\left(x_{j}, x_{i}\right)=<x_{j}, x_{i}>, f\left(x_{j}\right)=\sum_{i=1}^{n} a_{i} K\left(x_{j}, x_{i}\right), x_{\mathrm{i}}=$ (gender, age, race), and $\lambda_{i}\left(t \mid x_{i}\right)=\lambda_{0}(t) \exp \left(f\left(x_{i}\right)\right)$, which is the hazard function for Cox model where $t_{i}$ is lifetime of the $i$ th patient. Then we have to include the following equations to use the Newton-Raphson method:

$$
R=-\frac{1}{n} \sum_{i=1}^{n} a_{i}\left(\sum_{j=1}^{n} \delta_{j} K\left(x_{j}, x_{i}\right)\right)+\frac{1}{n} \sum_{i=1}^{n} \delta_{i} \ln \left\{B_{1(i)}\right\}+\xi\left(\sum_{i=1, i \neq p}^{n} a_{i}\left(\sum_{j=1}^{n} a_{j} K\left(x_{i}, x_{j}\right)\right)+a_{p} \sum_{j=1}^{n} a_{j} K\left(x_{p}, x_{j}\right)\right) .
$$

Using the partial derivatives obtained above, we have

$$
\frac{\partial R}{\partial a_{p}}=-\frac{1}{n} \sum_{j=1}^{n} \delta_{j} K\left(x_{j}, x_{p}\right)+\frac{1}{n} \sum_{i=1}^{n} \delta_{i} \frac{B_{2 p(i)}}{B_{1(i)}}+\xi\left(\sum_{i=1}^{n} a_{i} K\left(x_{i}, x_{p}\right)+\sum_{j=1}^{n} a_{j} K\left(x_{p}, x_{j}\right)\right),
$$

and 


$$
\begin{aligned}
\frac{\partial^{2} R}{\partial a_{p} \partial a_{q}}= & \frac{1}{n} \sum_{i=1}^{n} \delta_{i}\left(\frac{1}{B_{1(i)}} \sum_{j \in R_{i}} K\left(x_{j}, x_{p}\right) K\left(x_{j}, x_{q}\right) \exp \left(\sum_{k=1}^{n} a_{k} K\left(x_{j}, x_{k}\right)\right)\right. \\
& \left.-\frac{B_{2 p(i)} B_{2 q(i)}}{B^{2}{ }_{1(i)}}\right)+2 \xi K\left(x_{p}, x_{q}\right),
\end{aligned}
$$

where

$$
\begin{aligned}
& B_{1(i)}=\sum_{j \in R_{i}} \exp \left(\sum_{k=1}^{n} a_{k} K\left(x_{j}, x_{k}\right)\right), \quad R_{i}=\left\{j: t_{j} \geq t_{i}, j=1,2, \ldots, n\right\}, \\
& B_{2 p(i)}=\sum_{j \in R_{i}} K\left(x_{j}, x_{p}\right) \exp \left(\sum_{k=1}^{n} a_{k} K\left(x_{j}, x_{k}\right)\right), p=1,2, \ldots, n, \\
& B_{2 q(i)}=\sum_{j \in R_{i}} K\left(x_{j}, x_{q}\right) \exp \left(\sum_{k=1}^{n} a_{k} K\left(x_{j}, x_{k}\right)\right), q=1,2, \ldots, n .
\end{aligned}
$$

We used the kernel $K(x, y)=<C x, D y>$, where $\mathrm{C}$ and D are diagonal matrices (Manaf et al., 2011). We had verified the positive definiteness and symmetrical properties of kernel $K$. Our research and observations show that the greater the values of function $f(s)=\sum_{i=1}^{n} a_{i} K\left(s, s_{i}\right)$, the less chance of survival among the HIV patients chosen in random. This is shown in the following Figure 1.

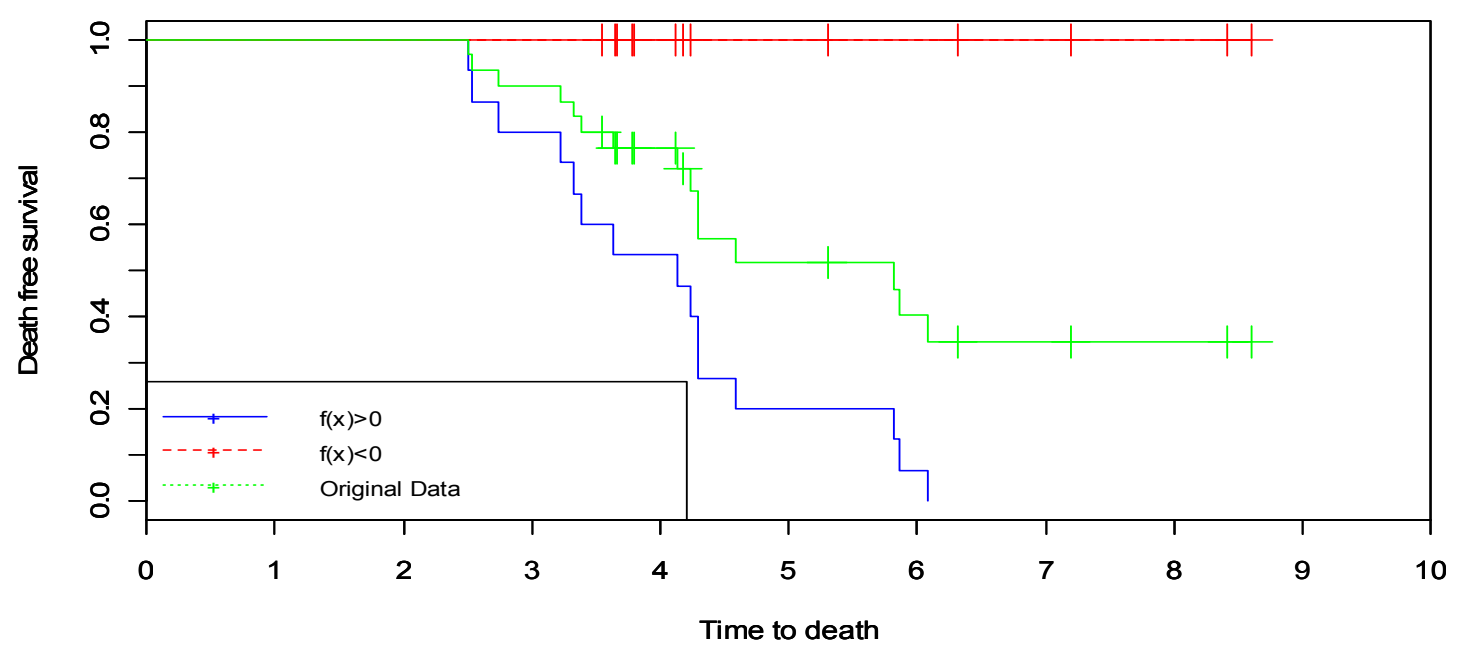

Figure 1. Survival of HIV patients

Using the Newton-Raphson method the optimal values of $a_{i}$ is obtained by setting the derivatives with respect to $a$ in $R$ to zero. We compared the result with the Gaussian Radial Basis function kernel, $K\left(x, x_{i}\right)=\exp \left(-\frac{1}{2}\left\langle x-x_{i}, x-x_{i}\right\rangle\right)$. Our result is shown in Table 1. 
Table 1. Results of Two Different Kernels

\begin{tabular}{|c|c|c|c|c|c|c|}
\hline & \multicolumn{3}{|c|}{$K\left(x, x_{i}\right)=\left\langle A x, B x_{i}\right\rangle$} & $K\left(x, x_{i}\right)=\exp \{-$ & \multicolumn{2}{|c|}{$-\frac{1}{2}\left\langle x_{i}-x, x_{i}-x\right\rangle$} \\
\hline$i$ & $a_{i}$ & $f\left(x_{i}\right)$ & $\exp \left(f\left(x_{i}\right)\right)$ & $a_{i}$ & $f\left(x_{i}\right)$ & $\exp \left(f\left(x_{i}\right)\right)$ \\
\hline 1 & $-6.571328 \mathrm{e}-03$ & 1.0803817 & 2.9458038 & 0.129739937 & 0.18785887 & 1.2066632 \\
\hline 2 & $3.818778 \mathrm{e}-04$ & 1.1058087 & 3.0216670 & 0.173914696 & 0.14436110 & 1.1553012 \\
\hline 3 & $-6.358875 e-04$ & 1.3355435 & 3.8020618 & 0.218131636 & 0.23427598 & 1.2639933 \\
\hline 4 & $-1.701724 \mathrm{e}-03$ & 1.5978747 & 4.9425169 & 0.116957073 & 0.15375643 & 1.1662068 \\
\hline 5 & $-3.052682 \mathrm{e}-03$ & 1.1776466 & 3.2467242 & 0.136644639 & 0.18991145 & 1.2091425 \\
\hline 6 & $3.269568 \mathrm{e}-03$ & 1.0421580 & 2.8353290 & 0.079175542 & 0.15908422 & 1.1724367 \\
\hline 7 & $4.034653 \mathrm{e}-03$ & 0.9161683 & 2.4996940 & 0.116962975 & 0.16858607 & 1.1836301 \\
\hline 8 & $2.396903 \mathrm{e}-03$ & 0.7243128 & 2.0633128 & 0.081691220 & 0.25496977 & 1.2904226 \\
\hline 9 & $2.878405 \mathrm{e}-03$ & 0.6907156 & 1.9951427 & 0.160807013 & 0.25418269 & 1.2894073 \\
\hline 10 & $7.937093 \mathrm{e}-04$ & 0.4774516 & 1.6119613 & 0.174749874 & 0.26432793 & 1.3025553 \\
\hline 11 & $2.369007 \mathrm{e}-03$ & 0.6303122 & 1.8781969 & 0.177146810 & 0.24826214 & 1.2817959 \\
\hline 12 & $2.997758 \mathrm{e}-03$ & 0.6639095 & 1.9423712 & 0.139188313 & 0.24145605 & 1.2731015 \\
\hline 13 & $-1.544913 e-03$ & 0.4408361 & 1.5540060 & 0.005409531 & 0.18045094 & 1.1977574 \\
\hline 14 & $7.542037 \mathrm{e}-03$ & 0.3090386 & 1.3621150 & 0.175677868 & 0.15535025 & 1.1680670 \\
\hline 15 & $-3.121462 \mathrm{e}-04$ & 0.4794866 & 1.6152450 & 0.138615666 & 0.19745615 & 1.2182996 \\
\hline 16 & $-8.216203 \mathrm{e}-04$ & -1.4328849 & 0.2386195 & -0.059706201 & -0.22576971 & 0.7979018 \\
\hline 17 & $-1.165802 \mathrm{e}-02$ & -1.2574178 & 0.2843874 & -0.176235680 & -0.33967263 & 0.7120034 \\
\hline 18 & $-8.956521 \mathrm{e}-03$ & -0.9429187 & 0.3894894 & -0.123189140 & -0.12618218 & 0.8814543 \\
\hline 19 & $-5.895857 \mathrm{e}-05$ & -0.1223129 & 0.8848715 & -0.152238094 & -0.04239335 & 0.9584927 \\
\hline 20 & $-2.303194 \mathrm{e}-03$ & -0.8080547 & 0.4457243 & -0.122136320 & -0.21545565 & 0.8061740 \\
\hline 21 & $1.335727 \mathrm{e}-03$ & -0.4900623 & 0.6125882 & -0.057326269 & -0.17656795 & 0.8381418 \\
\hline 22 & $-8.834102 \mathrm{e}-04$ & -0.2413472 & 0.7855688 & -0.398832658 & -0.43254441 & 0.6488560 \\
\hline 23 & $-3.405090 \mathrm{e}-03$ & -1.1368092 & 0.3208411 & -0.403182560 & -0.47979802 & 0.6189084 \\
\hline 24 & $-1.861149 \mathrm{e}-03$ & -1.0806055 & 0.3393900 & -0.127591108 & -0.32483308 & 0.7226480 \\
\hline 25 & $2.185279 \mathrm{e}-04$ & -0.4989532 & 0.6071659 & -0.045261685 & -0.08283722 & 0.9205010 \\
\hline 26 & $1.289077 \mathrm{e}-03$ & -1.1368092 & 0.3208411 & -0.024314446 & -0.47979802 & 0.6189084 \\
\hline 27 & $-4.513436 \mathrm{e}-03$ & -0.2686450 & 0.7644146 & -0.110502264 & -0.44325517 & 0.6419434 \\
\hline 28 & $2.688869 \mathrm{e}-02$ & -0.5399496 & 0.5827777 & -0.129127364 & -0.42241788 & 0.6554601 \\
\hline 29 & $1.314224 \mathrm{e}-03$ & -0.7378419 & 0.4781447 & -0.025176529 & -0.07878944 & 0.9242345 \\
\hline 30 & $4.379507 \mathrm{e}-03$ & -0.4496570 & 0.6378469 & -0.080555841 & -0.43651604 & 0.6462841 \\
\hline
\end{tabular}

\section{Conclusion}

Several other kernels can be generated and then applied to different sets of data. It is important that we are able to verify that the kernels fulfill all the rules and theories of RKHS. The derivatives used in this RKHS method are applicable to all kernels used to determine $f(x)$ in the Cox hazard function models. It should be noted that RKHS kernels can be used in models of several research areas such as in business, engineering and medical sciences because of the connection between data distributions and kernels. In fact, more researches can be performed to show that RKHS method can solve many other problems that involve mathematics and statistics. 


\section{References}

Aronszajn, N. (1950). Theory of Reproducing Kernels. Trans. American. Mathematical Soc., 686, $337-404$. http://dx.doi.org/10.1090/S0002-9947-1950-0051437-7

Berlinet, A., \& Thomas-Aqnan, C. (2003). Reproducing Kernel Hilbert Spaces in Probability and Statistics. Kluwer Academic, U.K.

Burbea, J. (1976). Total Positivity of Certain Reproducing Kernels. Pacific Journal of Mathematics, 67(1), 101-130. http://dx.doi.org/10.2140/pjm.1976.67.101

Hille, E. (1972). Introduction to General Theory of Reproducing Kernels. Rocky Mountain Journal of Mathematics, 2(3), 321-367. http://dx.doi.org/10.1216/RMJ-1972-2-3-321

Kimeldorf, G., \& Wahba (1971). Some results on Tchebycheffian spline functions. Journal of Mathematical Analysis and Applications, 33(1), 82-95. http://dx.doi.org/10.1016/0022-247X(71)90184-3

Li, H., \& Luan Y. (2003). Kernel Cox Regression Models for Linking Gene Expression Profiles to Censored Survival Data. Pacific Symposium on Bio-computing, 8, 65-76.

Manaf, N. A. A., Gafurjan, I., Bakar, A., Rizam, M., \& Aljawadi, B. (2011). Exploring Reproducing Kernel Hilbert Space and its Application to Survival Data. Australian Journal of Basic and Applied Sciences, 5(11), 1268-1274.

Wahba, G. (1990). SIAM, Philadelphia.

Wahba, G. (1998). Technical Report, UW Madison.

\section{Copyrights}

Copyright for this article is retained by the author(s), with first publication rights granted to the journal.

This is an open-access article distributed under the terms and conditions of the Creative Commons Attribution license (http://creativecommons.org/licenses/by/3.0/). 